\title{
PCR-BASED CONSTRUCTION AND TRANSFORMATION OF COdY GENE DELETION CONSTRUCT IN STREPTOCOCCUS PNEUMONIAE
}

\author{
Aisyah Mohamed Rehan ${ }^{1}$, Mohammad Izwan Enche Othman ${ }^{1}$, Nor Munirah Mohd Amin ${ }^{2}$, \\ Intan Azura Shahdan² Hanani Ahmad Yusof@Hanafi ${ }^{2}$ \\ ${ }^{1}$ Department of Biotechnology, Kulliyyah of Science, International Islamic University \\ Malaysia, Kuantan, Pahang, 'Department of Biomedical Science, Kulliyyah of Allied \\ Health Sciences, International Islamic University Malaysia, Kuantan, Pahang
}

Presenter: Mohammad Izwan Bin Enche Othman, izwan.en@live.iium.edu.my

Introduction: Streptococcus pneumoniae (S. pneumoniae) is a gram-positive diplococci belonging to the genus Streptococcus and it is a well-studied pathogenic bacterium. Pneumococcal diseases such as otitis media, pneumonia, sepsis and meningitis caused by pathogenic strains of $S$. pneumoniae still brought significant mortality and morbidity worldwide. The pathogenicity of S. pneumoniae is exerted by various virulence factors and one of it is the enzyme hyaluronate lyase. Hyaluronate lyase plays a major role in the invasive capability of S. pneumoniae. Its mechanism of action and crystallographic structure have been determinedbut its regulatory mechanism is still poorly understood. Drawing connections between the nutritional behaviour and invasive property of $S$. pneumoniae, CodY regulator is hypothesized as a potential hyaluronate lyase regulator. This work was aimed to construct CodY deficient mutant of S. pneumoniae to form foundational work for the study of CodY regulatory effect on hyaluronate lyase.

Materials and method: A single gene-deletion method was chosen to create CodY mutant construct containing aphA-3 gene (encoding kanamycin resistance protein) replacement cassette by employing recombinant fusion PCR method. A single band DNA product at the expected size of 2,563 bp after recombinant fusion PCR was visualized on agarose gel electrophoresis. This indicated that the linear recombinant amplicon has been successfully constructed. The recombinant amplicon was transformed into $S$. pneumoniae cells of Malaysian local clinical isolate by employing the competence stimulating peptide (CSP-1) to enhance and induce natural competence.

Results: No viable CodY mutant was evident post-transformation. The negative transformation result was postulated to be due to the essential nature of CodY regulator.

Conclusion: This work can provide basis for recombinant fusion PCR method in designing and constructing single gene deletion construct. However, further work is needed to identify the regulatory agent of hyaluronate lyase. 